\title{
Opuntia setocarpa, una especie nueva de nopal del occidente de México
}

\author{
Opuntia setocarpa, a new species of nopal from Western Mexico
}

\author{
Hilda Julieta Arreola-Nava ${ }^{a}$, Ramón Cuevas-Guzmán ${ }^{\text {b,* }}$, Luis Guzmán-Hernández ${ }^{\mathrm{b}}$ \\ y Adriana González-Durán ${ }^{\mathrm{a}}$ \\ a Departamento de Botánica y Zoología, Centro Universitario de Ciencias Biológicas y Agropecuarias, Universidad de Guadalajara, Km 15.5 Carr. \\ Guadalajara-Nogales, Apartado postal 139, 45110 Zapopan, Jalisco, México \\ ${ }^{\mathrm{b}}$ Instituto Manantlán de Ecología y Conservación de la Biodiversidad, Centro Universitario de la Costa Sur, Universidad de Guadalajara, Av. Independencia \\ Nacional 151, 48900 Autlán de Navarro, Jalisco, México \\ Recibido el 23 de octubre de 2016; aceptado el 11 de mayo de 2017 \\ Disponible en Internet el 23 de noviembre de 2017
}

\section{Resumen}

Se describe e ilustra Opuntia setocarpa, un nopal del estado de Jalisco. La especie pertenece a la serie Criniferae Pfeiffer. Se distingue por sus cladodios con epidermis papilosa, aréolas pequeñas y próximas, las aréolas de cladodios, pericarpelo y frutos con cerdas setosas largas y blancas, que las pierde en la madurez. Se proporciona información sobre su hábitat y se discuten sus relaciones con las especies más cercanas.

(C) 2017 Universidad Nacional Autónoma de México, Instituto de Biología. Este es un artículo Open Access bajo la licencia CC BY-NC-ND (http://creativecommons.org/licenses/by-nc-nd/4.0/).

Palabras clave: Cactaceae; Jalisco; Opuntia huajuapensis; Opuntia leucotricha; Opuntia pilifera; Serie Criniferae

\section{Abstract}

Opuntia setocarpa, a nopal from state of Jalisco is described and illustrated. The species belongs to the series $C$ riniferae Pfeiffer. It is distinguished by its cladodes with papillose epidermis, small and close together areoles, the areoles of cladodes, pericarpel and fruits with white long bristles, these becoming caducous. Information about its habitat is provided and its relationship with the closest species is discussed.

(C) 2017 Universidad Nacional Autónoma de México, Instituto de Biología. This is an open access article under the CC BY-NC-ND license (http://creativecommons.org/licenses/by-nc-nd/4.0/).

Keywords: Cactaceae; Jalisco; Opuntia huajuapensis; Opuntia leucotricha; Opuntia pilifera; Series Criniferae

\section{Introducción}

Cactaceae es una de las familias con mayor riqueza biológica en las zonas áridas, semiáridas, tropicales secas o lugares con clima fuertemente estacional (Hernández, Gómez-Hinostrosa y Goettsch-Cabello, 2004), y es de las pocas familias que cuentan con tratamiento taxonómico en México (Bravo-Hollis, 1978; Bravo-Hollis y Sánchez-Mejorada, 1991), país en el que han

\footnotetext{
* Autor para correspondencia.

Correo electrónico: rcuevas@ cucsur.udg.mx (R. Cuevas-Guzmán).

La revisión por pares es responsabilidad de la Universidad Nacional Autónoma de México.
}

alcanzado una diversificación extraordinaria. En Jalisco se han realizado inventarios taxonómicos de la familia (Arreola-Nava, 1990) y existe una guía para la identificación de Opuntia, el género mejor representado en esta entidad federativa, con 29 especies (González-Durán, Riojas-López y Arreola-Nava, 2001) y para el cual se siguen adicionando nuevas especies (Hernández, Gómez-Hinostrosa y Bárcenas, 2001; Pérez, Reyes y Brachet, 2005; Scheinvar y Manzanero, 2009; Scheinvar y RodríguezFuentes, 2003). Se encontró en el estado de Jalisco una especie que por sus características morfológicas no encuentra acomodo en las especies conocidas, por lo cual se propone como nueva para la ciencia. 


\section{Materiales y métodos}

En la transición entre el bosque tropical caducifolio y el bosque de Quercus, en bosque de Pinus-Quercus y en ecotonos entre bosque de Quercus y el bosque mesófilo de montaña, en la sierra de Cacoma se recolectaron ejemplares de Opuntia, los cuales fueron deshidratados, etiquetados y montados para después depositarse en el Herbario ZEA del Centro Universitario de la Costa Sur (CU Costa Sur) de la Universidad de Guadalajara. Los datos de altura y diámetro en la base de los individuos se registraron directamente en campo, además de tomar fotografías de las partes vegetativas, flores y frutos utilizando una cámara fotográfica Canon EOS 7D. Se hizo una revisión minuciosa del material vegetativo y reproductivo de la Opuntia para su determinación y descripción, haciendo observaciones detalladas al microscopio Zeiss Stemi 2000 8-50× y revisando la literatura especializada. Para la determinación de los grados Brix se utilizó el extracto de la pulpa de frutos persistentes, la cual se colocó en un refractómetro marca HANNA, modelo HI 96801. Se concluye que el taxón estudiado pertenece a una especie aún no clasificada, por lo cual se propone como nueva para la ciencia. Se elaboró un dibujo a tinta china utilizando ejemplares recolectados en campo, con apoyo de fotografías y observaciones de las estructuras en el estereoscopio.

\section{Descripción}

Opuntia setocarpa Arreola-Nava, Guzm.-Hern. et Cuevas sp. nov. (figs. 1-2)

Tipo: México. Jalisco: municipio de Autlán de Navarro, cerca de las Neverías en la sierra de Cacoma, $19^{\circ} 50^{\prime} 51.2^{\prime \prime} \mathrm{N}$, $104^{\circ} 27^{\prime} 5.5^{\prime \prime}$ O, bosque de Pinus-Quercus, 2,144 m, 17 marzo 2007 (fl), R. Cuevas y L. Guzmán 9022 (holotipo: ZEA; isotipos: IBUG, IEB, MEXU).

Fruticosa vel arborea 0.6-3 m alta; articuli papillosi late ovali vel orbiculari $16-40 \mathrm{~cm}$ longi, $11-33 \mathrm{~cm}$ lati; areolae intrer sese 11-20 mm distantes, spinae 4-7 acicularia vel subulatiae; flores luti 5-6 cm longi, hypanthium $2.8-3.4 \mathrm{~cm}$ longum, setae albae $4-10$ areolis $1.6-3.5 \mathrm{~cm}$ longae; bacca luteo-viridis 3.2 $4.6 \mathrm{~cm}$ longa; semina reniformia vel orbiculatia $2.7-3.1 \mathrm{~mm}$ longa.

Arbustiva o arborescente, 0.6-3 $\mathrm{m}$ de altura. Tronco de 5$38 \mathrm{~cm}$ de largo, 8-21 cm de diámetro, escamoso, con frecuencia con algunos grupos de aréolas dispersos, las aréolas de la estación con 16-28 espinas blancas a amarillas de (1.3) $2.5-3 \mathrm{~cm}$ de largo, las espinas de las estaciones anteriores tornándose moreno oscuro. Artículos anchamente ovales a orbiculares, $16-40 \mathrm{~cm}$ de largo, $11-33 \mathrm{~cm}$ de ancho, epidermis papilosa de color verde pálido a amarillento en cladodios maduros y verde brillante en cladodios jóvenes y algunos maduros; aréolas dispuestas en 12 16 series de espirales, elípticas a casi orbiculares, 2.1-4.1 mm de largo, 1.2-2.9 mm de ancho, distantes entre sí 11-20 mm, con fieltro blanco en el borde, el cual se torna gris con la edad, glóquidas hasta de $3 \mathrm{~mm}$ de largo, amarillas, situadas en la parte superior de las aréolas; hojas subuladas de 2-5 mm de largo, rojizas, evidentes en cladodios jóvenes; espinas 4-7, aciculares a subuladas, divergentes, $0.4-1.7 \mathrm{~cm}$ de largo, amarillo pálidas, con la edad una o dos de ellas blancas, las inferiores más largas y fuertes que el resto y adpresas o reflejas, en la base de la aréola 4 - 10 cerdas blancas, rectas, onduladas o inflexas, pronto caedizas, aunque algunas persistentes y tornándose negras, $1.3-2.4 \mathrm{~cm}$ de largo. Flores campanuladas, $5-6.5 \mathrm{~cm}$ de largo, $3.4-6.9 \mathrm{~cm}$ de diámetro en la antesis; pericarpelo de 2.8-3.4 cm de largo, 2.0$2.5 \mathrm{~cm}$ de diámetro, con aréolas distantes entre sí de 1.7-2.1 mm, 1.2-1.6 mm de diámetro, con lana color castaño, con glóquidas cortas, amarillo pálido y 4-10 cerdas blancas, rectas, onduladas a inflexas, $1.6-3.5 \mathrm{~cm}$ de largo, caducas con el tiempo; tépalos externos, obovados, truncados u oblanceolados, $1.7-4 \mathrm{~cm}$ de largo, verde amarillentos, con una mácula rosa y mucronados en el ápice; tépalos internos obovados o truncados, amarillos, todos los tépalos tornándose rosas con la edad; estambres $1.5-1.7 \mathrm{~cm}$ de largo, filamentos blancos, anteras de $2 \mathrm{~mm}$ de largo; estilo 1.4-1.6 cm de largo, blanco, estigma con 6-12 lóbulos, verdes. Fruto subgloboso a turbinado, $3.2-4.6 \mathrm{~cm}$ de largo, $2.4-3.2 \mathrm{~cm}$ de diámetro, epidermis finamente papilosa, amarillo-verdosa; aréolas circulares de 1.1-2.1 mm de diámetro, con fieltro amarillo o blanquecino, con glóquidas cortas de color amarrillo pálido y 4-10 cerdas blancas, rectas, onduladas o inflexas, pronto caedizas, aunque algunas persistentes y tornándose negras, $1.3-2 \mathrm{~cm}$ de largo; parénquima del pericarpelo muy desarrollado, pulpa blanco verdosa, de sabor dulce con $10.7-11.2^{\circ} \mathrm{Bx}$ a $23.5^{\circ} \mathrm{C}$, en los frutos persistentes; semillas reniformes a orbiculares, 2.7$3.1 \mathrm{~mm}$ de largo, 2.1-2.5 mm de ancho, gris a castaño claro, con un arilo membranoso, testa de la epidermis finamente tuberculada.

\section{Resumen taxonómico}

Ejemplares adicionales. México. Jalisco: municipio de Autlán de Navarro, 5-6 km adelante de El Jalocote, camino a San Juan Cacoma, 1,965 m, 5 marzo 2004 (fl y fr), R. Cuevas y L. Guzmán 8482 (ZEA); $4.5 \mathrm{~km}$ adelante de El Jalocote, camino a San Juan Cacoma, 1,950 m, 5 marzo 2004 (fl), R. Cuevas y E. Sánchez 8491 (ZEA); 1,840 m, 26 marzo 2004 (fl), R. Cuevas y L. Guzmán 8502 (ZEA); 3 noviembre 2007 (fr), H. Arreola y L. Guzmán 1673 (IBUG y ZEA); cerca de las neverías en la sierra de Cacoma, 2,144 m, 17 marzo 2007 (fl), R. Cuevas y L. Guzmán 9023, 9024 (ZEA). Municipio de Ameca, cerro Grande de Ameca, acceso por la carretera Ahualulco-Ameca, 2,500 m, 28 noviembre 2004 (fr), A. Machuca 9638 (IBUG).

Distribución y hábitat. La especie se conoce solo en los municipios de Autlán de Navarro en la sierra de Cacoma y cerro Grande en el municipio de Ameca en el estado de Jalisco, entre los 1,800-2,500 m de elevación. Se ha recolectado y observado en la transición entre bosque tropical caducifolio y el bosque de Quercus, donde convive con Agave maximiliana Hort., Arbutus tessellata Sorensen, Comarostaphylis glaucescens Zucc. ex Klotzsch, Lysiloma acapulcensis Benth., Mammillaria scrippsiana (Britton y Rose) Orcutt, Mimosa galeottii Benth., Pittocaulon velatum (Greenm.) $\mathrm{H}$. Rob. y Brettell, Quercus resinosa Liebm., Salvia lavanduloides Kunth, Senecio stoechadiformis DC. y Wigandia caracasana Kunth. En ocasiones penetra en los ecotonos entre bosque de 


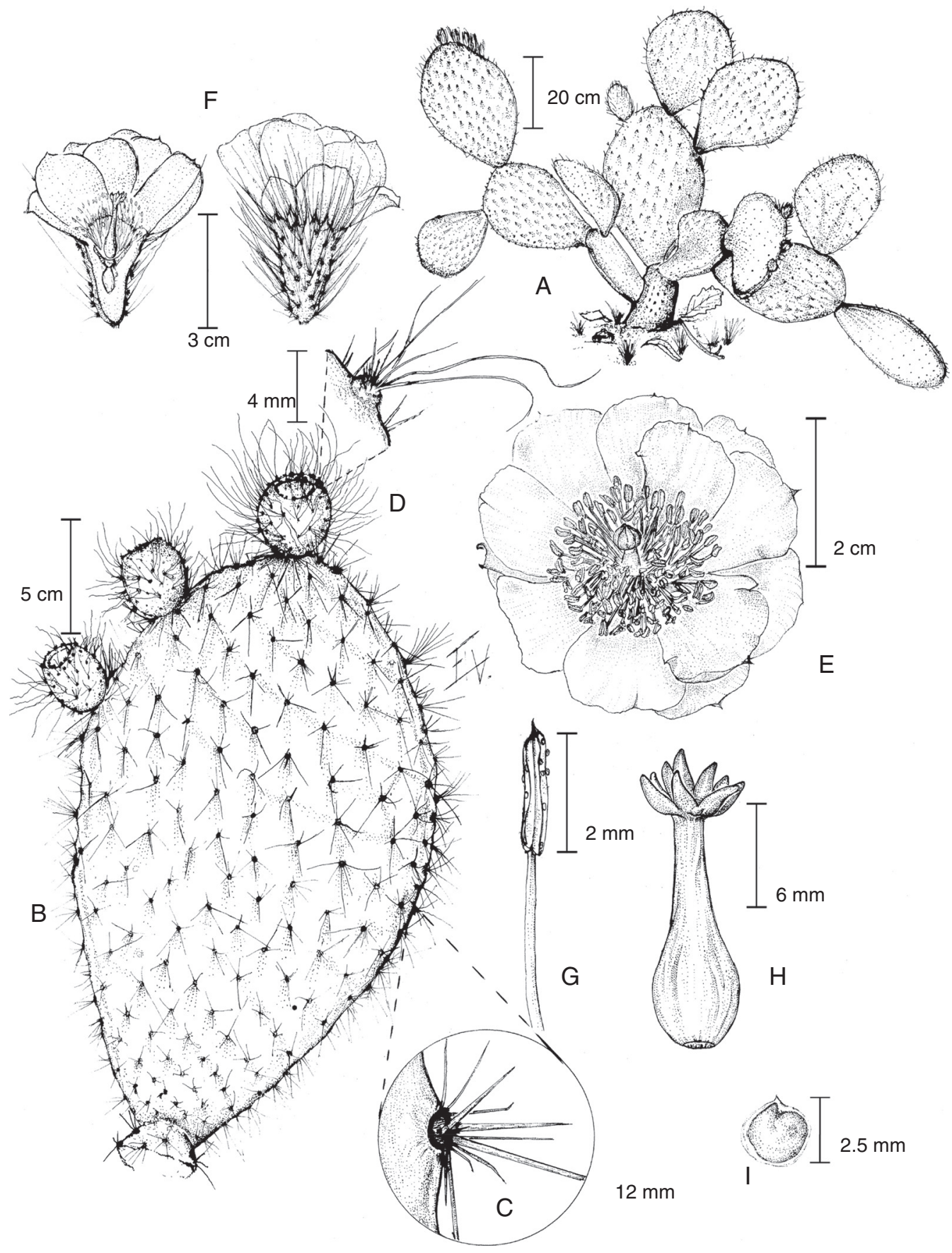

Figura 1. Opuntia setocarpa. A: hábito; B: cladodio con frutos aún con cerdas; C: detalle de una aréola del cladodio con espinas y cerdas; D: detalle de una aréola del fruto; E: detalle de una vista superior de la flor; F: corte longitudinal de una flor; G: detalle de un estambre; H: detalle del pistilo; I: detalle de una semilla. Flores con base en Cuevas y Guzmán 9022; cladodio, frutos y semilla con base en Cuevas y Sánchez 8491.

Quercus y bosque mesófilo de montaña y entonces se le observa con Bocconia arborea S. Watson, Ficus sp., Fraxinus uhdei (Wenzig) Lingelsh., Litsea glaucescens Kunth, Lysiloma divaricatum (Jacq.) J.F. Macbr., Quercus magnoliifolia Née, Solanum edmundoi Cuevas y Núñez y Vitis blancoi Munson. También se le ha recolectado en bosque de Pinus-Quercus con Arbutus tesellata, Juniperus flaccida Schltdl., Pinus lumholtzii B.L. Rob. y Fernald, P. oocarpa Schiede ex Schltdl., Quercus castanea Née y $Q$. obtusata Humb. y Bonpl. Con frecuencia se han observado semillas de este nopal en las excretas de la zorra gris, Urocyon cinereoargenteus, la cual parece ser uno de sus dispersores.

Fenología. Como sucede con la mayor parte del género Opuntia, la especie florece en marzo y abril y fructifica de julio a noviembre; en ocasiones se encuentran algunos frutos que persisten hasta abril.

Etimología. El nombre de la especie hace alusión a la abundancia de cerdas largas y blancas que se presentan en las aréolas del pericarpelo, aunque en el fruto (carpus) las cerdas largas son caedizas con el tiempo. 


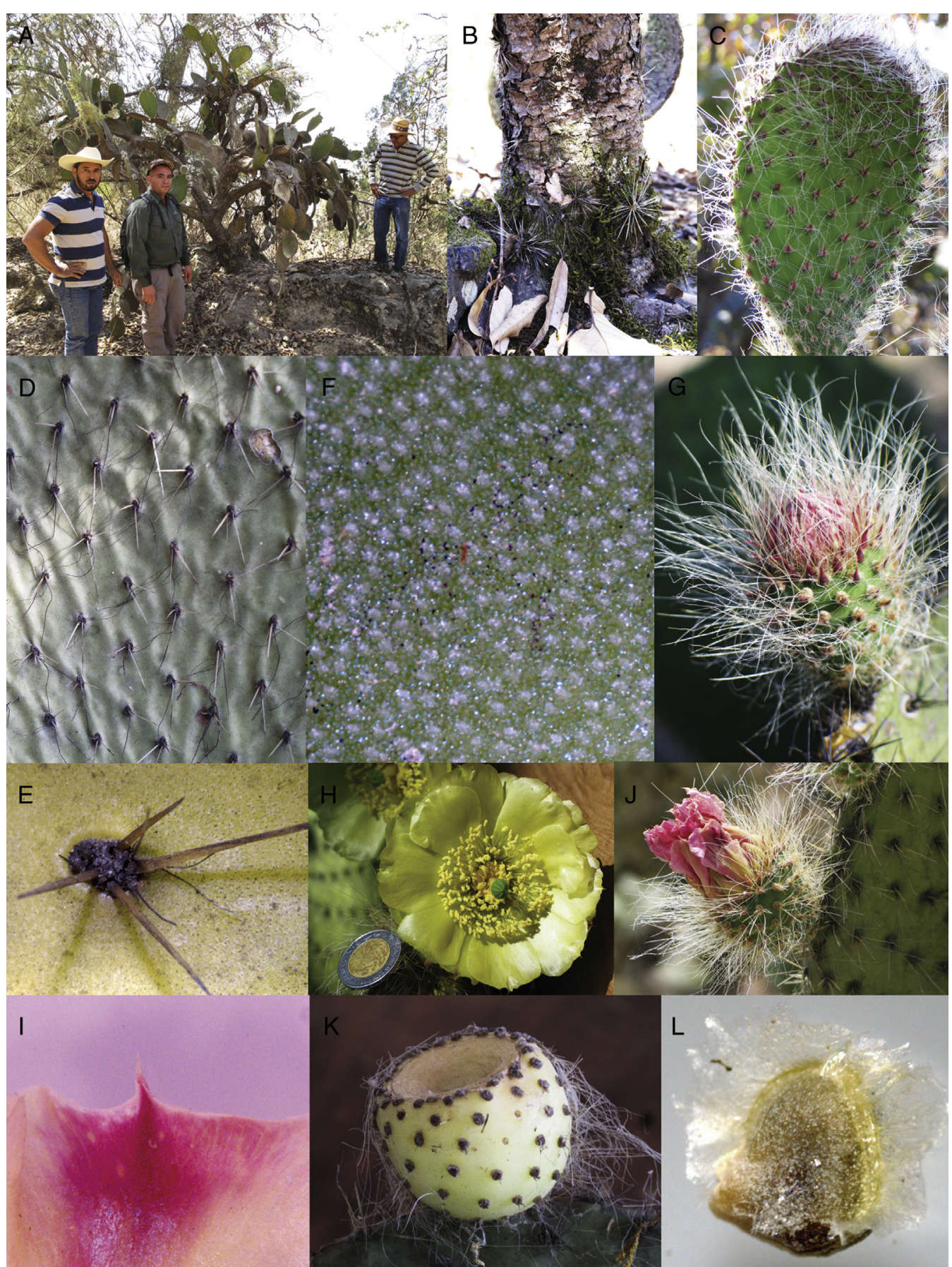

Figura 2. Opuntia setocarpa. A: hábito arborescente; B: tronco escamoso con aréolas con muchas espinas; C: cladodio joven con aréolas, glóquidas, hojas y cerdas; D: cladodio desarrollado con espinas y cerdas negras persistentes; E: acercamiento de una aréola con espinas y algunas cerdas persistentes $(6.5 \times)$; F: epidermis papilosa de un cladodio $(20 \times)$; G: botón floral con aréolas, glóquidas, hojas y cerdas; $\mathrm{H}$ : flor abierta en la que se observan tépalos interiores, estambres y estigmas; I: tépalo por la parte exterior mostrando la parte rosa y el apículo $(6.5 \times)$; J: flor después de la antesis; K: fruto con aréolas, glóquidas y parte de él aún con cerdas; L: semilla con arilo membranáceo y testa finamente tuberculada $(12.5 \times)$.

Estado de conservación. La especie es abundante localmente, hay buena reproducción, ya que se observan individuos en todos los estadios de desarrollo, incluyendo plántulas y juveniles, además de que se desarrolla sobre grandes rocas que la protegen, donde forma colonias, sin problema de supervivencia.

\section{Comentarios taxonómicos}

Por sus cladodios con epidermis papilosa y la presencia de pelos largos y abundantes en las aréolas, Opuntia setocarpa encuentra acomodo en la serie Criniferae (Bravo-Hollis, 1978). 
Tabla 1

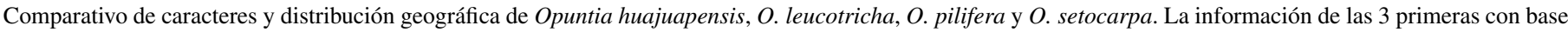
en Bravo-Hollis (1978) y Anderson (2001); la de O. setocarpa, con base en este trabajo.

\begin{tabular}{|c|c|c|c|c|}
\hline Carácter & O. huajuapensis & O. leucotricha & O. pilifera & O. setocarpa \\
\hline Hábito & Arbóreo y arbustivo & Arborescente & Arborescente & Arbustivo a arborescente \\
\hline Tronco & $\begin{array}{l}\text { Conspicuo, hasta } 1 \mathrm{~m} \text { de } \\
\text { longitud cuando arbórea }\end{array}$ & Bien definido, escamoso & $\begin{array}{l}\text { Bien definido, grueso y } \\
\text { leñoso }\end{array}$ & Bien definido, escamoso \\
\hline Cladodios, forma & Obovados a orbiculares & Oblongos a orbiculares & Oblongos a orbiculares & $\begin{array}{l}\text { Anchamente ovales a } \\
\text { orbiculares }\end{array}$ \\
\hline Epidermis & $\begin{array}{l}\text { Pubescente, sin brillo, } \\
\text { tricomas largos }\end{array}$ & Pubescente & Glabra & Papilosa \\
\hline Aréolas, series & $\begin{array}{l}15-16(-19), \text { distantes } \\
20-30 \mathrm{~mm}\end{array}$ & 18-20, distantes 8-15 mm & Distantes 20-30 mm & 12-16, distantes $11-20 \mathrm{~mm}$ \\
\hline Aréolas, color del fieltro & Gris oscuro & Blanco & Negruzco & Blanco, gris con la edad \\
\hline Cerdas & $4-10$, rectas, blancas & $1-2$, retorcidas & $\begin{array}{l}\text { Blanco sedosas, abundantes } \\
\text { en artículos jóvenes }\end{array}$ & $\begin{array}{l}\text { 4-10, rectas, onduladas a } \\
\text { inflexas }\end{array}$ \\
\hline Color de espinas & $\begin{array}{l}\text { Amarillo claro en la base y en } \\
\text { el ápice más oscuras }\end{array}$ & $\begin{array}{l}\text { Amarillo con punta } \\
\text { translúcida, blanco con la } \\
\text { edad }\end{array}$ & $\begin{array}{l}\text { Amarillo a blanquecino, } \\
\text { negro con la edad }\end{array}$ & $\begin{array}{l}\text { Amarillo pálido, blanco con } \\
\text { la edad }\end{array}$ \\
\hline Color de flores & $\begin{array}{l}\text { Amarillo, con ápices } \\
\text { anaranjados }\end{array}$ & $\begin{array}{l}\text { Amarillo-verdoso, con } \\
\text { manchas rojizas, tornándose } \\
\text { rosadas }\end{array}$ & Rosa a rojo-púrpura & $\begin{array}{l}\text { Amarillo con los tépalos } \\
\text { externos rosa en su ápice, } \\
\text { tornándose rosado }\end{array}$ \\
\hline Pericarpelo & $\begin{array}{l}\text { Aréolas con numerosas } \\
\text { cerdas hasta de } 3 \mathrm{~cm} \text { de largo }\end{array}$ & $\begin{array}{l}\text { Aréolas con cerdas hasta de } \\
1 \mathrm{~cm} \text { de largo }\end{array}$ & $\begin{array}{l}\text { Aréolas con glóquidas } \\
\text { morenas y pelos blancos } \\
\text { caducos }\end{array}$ & $\begin{array}{l}\text { Areolas con } 4-10 \text { cerdas } \\
\text { blancas de } 1.6-3.5 \mathrm{~cm} \text { de largo }\end{array}$ \\
\hline $\begin{array}{l}\text { Color del ápice de los } \\
\text { segmentos exteriores }\end{array}$ & Rojo & Rojizo & Tinto & Rosado \\
\hline Filamentos & Blanco a verde claro & Blanco & Rojizo & Blanco \\
\hline $\begin{array}{l}\text { Lóbulos del estigma, número } \\
\text { y color }\end{array}$ & $5-9$, blanco a verde claro & 6 -10, verde esmeralda & 6-7, verde claro & $6-12$, verde \\
\hline $\begin{array}{l}\text { Frutos, forma, largo }(\mathrm{cm}) \mathrm{y} \\
\text { color }\end{array}$ & Globoso, 3-4.3, amarillo & $\begin{array}{l}\text { Globoso, 4-6, blanco hasta } \\
\text { púrpura }\end{array}$ & Globoso, 3-5, rojo & $\begin{array}{l}\text { Subgloboso a turbinado, } \\
\text { 3.2-4.6, amarillo-verdoso }\end{array}$ \\
\hline Semillas & $\begin{array}{l}\text { Elípticas a subglobosas, con } \\
\text { tricomas en la parte media }\end{array}$ & $\begin{array}{l}\text { Orbiculares a elipsoides, con } \\
\text { tricomas en la superficie }\end{array}$ & Información no disponible & $\begin{array}{l}\text { Reniformes a orbiculares de } \\
\text { epidermis finamente tuberosa }\end{array}$ \\
\hline $\begin{array}{l}\text { Distribución geográfica } \\
\text { conocida }\end{array}$ & Oaxaca & $\begin{array}{l}\text { Durango, Guanajuato, } \\
\text { Hidalgo, Querétaro, San Luis } \\
\text { Potosí, Zacatecas }\end{array}$ & Oaxaca, Puebla, Tlaxcala & Jalisco \\
\hline
\end{tabular}

Tabla modificada de Scheinvar et al. (2015).

Opuntia huajuapensis Bravo, O. leucotricha DC. y O.pilifera F.A.C. Weber son las especies con las cuales parece tener mayor relación. De la primera se diferencia por sus troncos cortos de no más de $40 \mathrm{~cm}$ de longitud, la epidermis de los cladodios papilosa vs. pubescente y con tricomas largos, las aréolas de los cladodios más próximas entre sí (11-20 mm vs. $20-30 \mathrm{~mm}$ ) y las cerdas de las aréolas de los cladodios son al menos del doble de la longitud que las de O. huajuapensis. De O. leucotrica se separa por la presencia en el tallo de aréolas con numerosas espinas vs. pelos largos, setosos y flexibles hasta de $8 \mathrm{~cm}$ de largo; epidermis papilosa vs. pubescente; estilos blancos vs. rojos; fruto amarillo verdoso de epidermis finamente papilosa vs. blanco a púrpura y de epidermis pubescente; semillas finamente tuberculadas vs. con tricomas. De O. pilifera se diferencia por los cladodios con epidermis papilosa vs. glabra; flores amarillas vs. rosas a rojo púrpura; frutos amarillos vs. rojos; filamentos blancos vs. rojizos; estilos blancos vs. rojos y frutos amarillo verdosos vs. rojos. Opuntia perotensis se describió recientemente, y aunque podría tratarse de un híbrido (Scheinvar, Olalde-Parra y Gallegos-Vázquez, 2015), O. setocarpa podría separarse de ella por un tallo corto vs. ausente; cerdas blancas vs. de color marrón amarillas, rojizas a marrón; espinas amarillo pálidas a blancas con la edad vs. rojizas a marrón; pericarpelo con cerdas blancas de $0.5-2 \mathrm{~cm}$ de largo vs. cerdas amarillas y por lo general menores de $1 \mathrm{~cm}$; semillas con un arilo membranáceo vs. arilo de tricomas largos. Ecológicamente, O. setocarpa también difiere de las otras especies por su hábitat rupícola y las comunidades vegetales en que se le encuentra. Otros caracteres que separan a las especies se consignan en la tabla 1.

\section{Agradecimientos}

A Christine Niezgoda del Field Museum of Natural History de Chicago por el envío de literatura sobre el género Opuntia. El trabajo forma parte del proyecto «Inventario florístico de la Sierra de Manantlán y la costa sur de Jalisco» y fue apoyado económicamente a través del fondo de investigación del CU Costa Sur. Se agradece el apoyo en el trabajo de campo al M.C. José Guadalupe Morales Arias y Enrique V. Sánchez Rodríguez, este último también elaboró la ilustración. Al Ing. José Antonio Machuca explorador y recolector jalisciense que encontró de forma simultánea esta especie y la entregó a Adriana González Durán para su identificación. El Dr. Jesús Juan Rosales Adame apoyó en la determinación del concentrado de azúcar en los frutos. A Alba Cuevas Núñez por la edición de las figuras. 


\section{Referencias}

Anderson, E. F. (2001). The cactus family. Portland: Timber Press, Inc.

Arreola-Nava, H. J. (1990). Inventario de las cactáceas de Jalisco y su distribución. Cactáceas y Suculentas Mexicanas, 35, 3-12.

Bravo-Hollis, H. (1978). Las cactáceas de México, Segunda edición. México D.F.: Universidad Nacional Autónoma de México.

Bravo-Hollis, H. y Sánchez-Mejorada, H. (1991). Las cactáceas de México, Segunda edición. México D.F.: Universidad Nacional Autónoma de México.

González-Durán, D. A., Riojas-López, M. E. y Arreola-Nava, H. J. (2001). El género Opuntia en Jalisco: guía de campo. Guadalajara, Jalisco: Universidad de Guadalajara y Comisión Nacional para el Conocimiento y Uso de la Biodiversidad.

Hernández, H. M., Gómez-Hinostrosa, C. y Bárcenas, R. T. (2001). Studies on Mexican Cactaceae. I. Opuntia pachyrrhiza, a new species from the Chihuahuan Desert, Mexico. Novon, 11, 309-314.
Hernández, H. M., Gómez-Hinostrosa, C. y Goettsch-Cabello, B. (2004). Cactáceas. En A. J. García-Mendoza, M. J. Ordóñez, y M. Briones-Salas (Eds.), Biodiversidad de Oaxaca (pp. 199-207). México D.F.: Instituto de Biología, Universidad Nacional Autónoma de México/Fondo Oaxaqueño para la Conservación de la Naturaleza/World Wildlife Fund.

Pérez, C., Reyes, J. y Brachet, F. (2005). Opuntia olmeca, una nueva especie de la familia Cactaceae para el estado de Oaxaca, México. Cactáceas y Suculentas Mexicanas, 50, 89-95.

Scheinvar, L. y Manzanero, G. (2009). Opuntia chiangiana, una nueva especie de Cactaceae de Oaxaca, México. Novon, 19, 222-228.

Scheinvar, L., Olalde-Parra, G. y Gallegos-Vázquez, C. (2015). Una nueva especie del género Opuntia (Cactaceae) para el estado de Veracruz, México. Botanical Sciences, 93, 1-8.

Scheinvar, L. y Rodríguez-Fuentes, A. (2003). Nueva subespecie de Opuntia streptacantha (Cactaceae) de la altiplanicie mexicana. Anales del Instituto de Biología, Universidad Nacional Autónoma de México, Serie Botánica, 74, 303-311. 